\title{
Radially polarized Bessel-Gauss beams in ABCD optical systems and fiber-based generation
}

\author{
D. N. Schimpf,${ }^{1,3}$ W. P. Putnam, ${ }^{1,3}$ M. D. W. Grogan, ${ }^{2}$ S. Ramachandran, ${ }^{2}$ and F. X. Kärtner ${ }^{1,3}$ \\ ${ }^{\prime}$ Department of Electrical Engineering and Computer Science and Research Laboratory of Electronics \\ Massachusetts Institute of Technology, 77 Massachusetts Ave., Cambridge, Massachusetts 02139, USA \\ ${ }^{2}$ Photonics Center, Department of Electrical and Computer Engineering, Boston University, 8 Saint Mary's Street, Boston, MA 02215, USA \\ ${ }^{3}$ Center for Free-Electron Laser Science, DESY and Department of Physics, University of Hamburg, Notkestra $\square$ e 85, Hamburg 22607, Germany \\ Author e-mail address: dschimpf@mit.edu
}

\begin{abstract}
We derive solutions for radially polarized Bessel-Gauss beams in ABCD optical systems by superimposing decentered Gaussian beams with linear polarization states. We experimentally confirm the expression by employing a fiber-based mode-converter.

OCIS codes: (140.3300) Laser beam shaping; (220.4830) Systems design; (060.2310) Fiber optics;
\end{abstract}

Bessel-Gauss beams offer an extended depth of field in the focus as compared to conventional Gaussian beams. Furthermore, the radial polarization offers several interesting ramifications, such as (a) focusing to tighter spot-sizes [1], (b) mapping of the orientations of single molecules in three dimensions [2], (c) vacuum laser acceleration of electrons [3], (d) optical tweezers for stable 3-D trapping of metallic particles [4].

In this contribution, we derive solutions for radially polarized Bessel-Gauss beams in an intuitive way by superimposing decentered Gaussian beams with linear polarization states. The superposition results in expressions for the propagation of radially polarized Bessel-Gauss beams through ABCD optical systems hitherto not reported to the best of our knowledge. We find these expressions useful for the setup of optical systems using radially polarized beams.

Fig. 1 (a) illustrates the concept of the ray bundle. We form the radially polarized Bessel-Gauss beams by superimposing the component beams with linear polarization states such that their polarizations point in the radial direction. Furthermore, their beam centers are placed on a circle with radius $r_{\alpha 0}$ in the x-y plane and their beam directions point to the apex of a cone with a semi-aperture angle $\varepsilon_{0}[5]$. The advantage of this approach is that the transformation of the component beams in ABCD optical systems is known. Specifically, the dencentered Gaussian beams follow the trajectories of meridional rays, and their Gaussian beam parameters transform like that of conventional Gaussian beams. At the end of the propagation through the ABCD optical system, the dencentered Gaussians can be superimposed to form the radially polarized Bessel-Gauss beams.

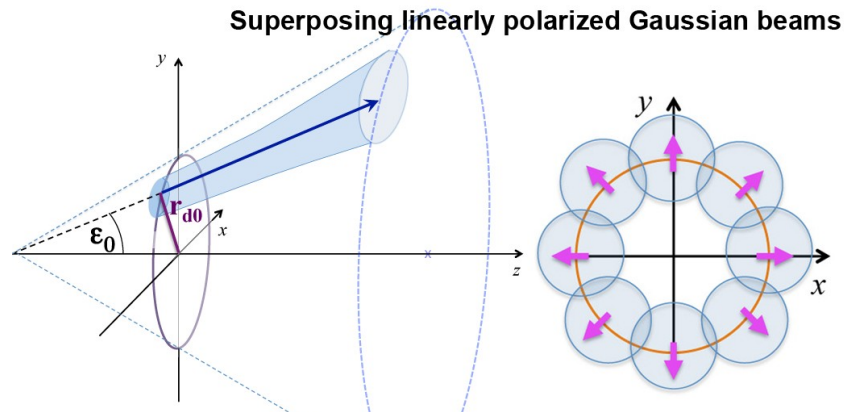

(a)

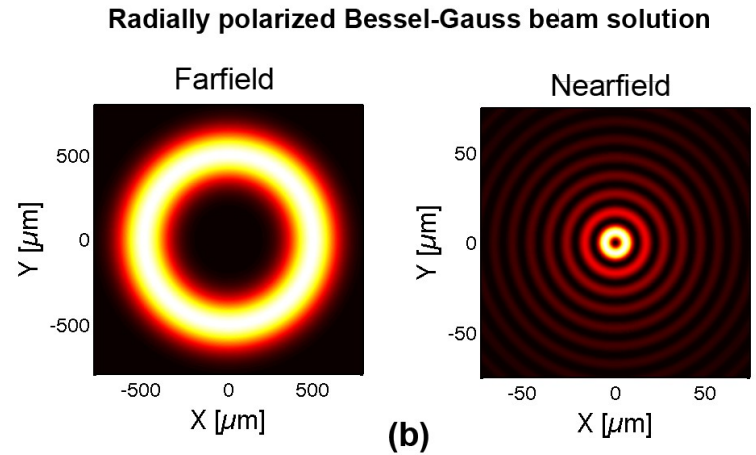

(b)

Fig. 1.: (a) Concept of superposing decentered Gaussian beams with linear polarization on a cone to radially polarized Bessel-Gauss beams; (b) resulting solution for radially polarized Bessel-Gauss beams

The radially polarized Bessel-Gauss beam at the final stage of the ABCD optical systems is expressed as

$$
\mathbf{A}(r, \theta, z) \propto \hat{\mathbf{r}} e^{i\left(k \cdot z-\omega_{0} \cdot t\right)} \cdot e^{i \psi} \cdot \frac{1}{w} \cdot e^{-i \phi} \cdot e^{i \frac{k}{2 q}\left(r^{2}+r_{d}^{2}\right)} \cdot J_{1}\left(k r\left(\varepsilon-\frac{r_{d}}{q}\right)\right)
$$


where $r_{d}$ is the final circle radius , $\varepsilon$ is the final semi aperture angle, and $\psi$ is a z-dependent phase-shift, $q$ is the Gaussian beam parameter and $\phi$ is the Gouy phase. These parameters are related to the initial ones via the components of the $\mathrm{ABCD}$ ray matrix of the optical system:

$$
\begin{aligned}
r_{d} & =A r_{d 0}+B \varepsilon_{0} & & =\frac{A q_{0}+B}{C q_{0}+D} \\
\varepsilon & =C r_{d 0}+D \varepsilon_{0} & & \\
\psi & =-\frac{k}{2}\left[C r_{d 0} r_{d}+B \varepsilon_{0} \varepsilon\right] & & =\arg \left(A+B / q_{0}\right)
\end{aligned}
$$

The solution for a radially polarized Bessel-Gauss beam is plotted for the case of free-space propagation in Fig. 1 (b). The nearfield is described by $\left(\mathrm{r}_{\mathrm{d} 0}=0, \varepsilon_{0}=3^{\circ}\right.$, waist $\left.\mathrm{w}_{0}=200 \mu \mathrm{m}, \lambda=1050 \mathrm{~nm}\right)$ and in the farfield is evaluated at $\mathrm{z}=10 \mathrm{~mm}$. It can be seen that the transverse field of the near field doesn't show an on-axis intensity - this is unlike the case of a Bessel-Gauss beam with linear polarization.

To experimentally confirm the form of the solution, we employ a fiber-based approach to produce radially polarized light. The output of a fiber-pigtailed laser diode $(\lambda=1050 \mathrm{~nm})$ is launched into a mode converter (in form of a longperiod grating) which is inscribed in a specialty optical fiber [6]. The output of the fiber is a radially polarized TM01 mode exhibiting a ring-like mode shape. On the backside of an axicon (fused silica, base angle $1^{\circ}$ ), the ring mode is magnified to a radius of $\sim 2.3 \mathrm{~mm}$ with the annulus showing a waist of $\sim 1 \mathrm{~mm}$. The axicon produces a phase such that the ring beam conically converges to an on-axis crossing point. At the corresponding plane we observe the radially polarized Bessel-Gauss beam. It is noteworthy that the ring-shaped radially polarized mode avoids the round tip of the axicon. Under conventional Gaussian-like illumination of an axicon, this round axicon tip causes unwanted interference effects. We completely eliminate these effects by using ring beam excitation. Therefore, the present approach results in high-quality Bessel-Gauss beam generation, as can be seen in Fig.2 (b), which compares experimental to the theoretical expression (Eq.1).

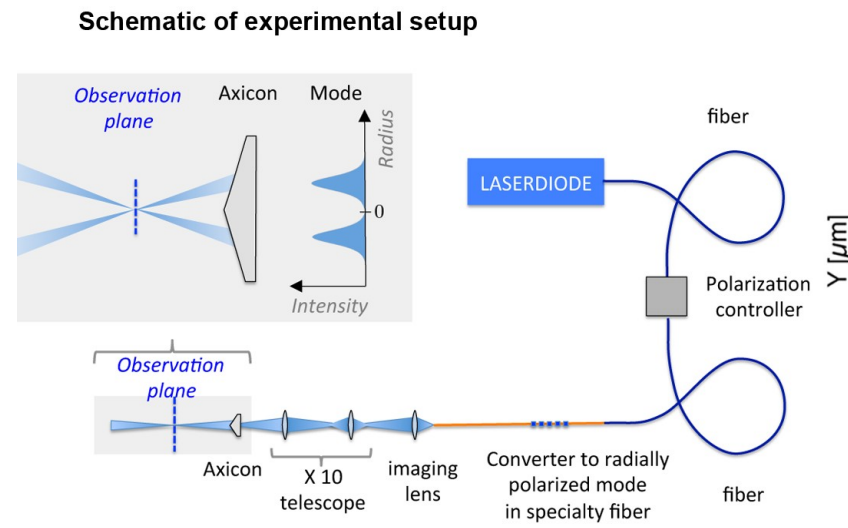

(a)
Experimental results
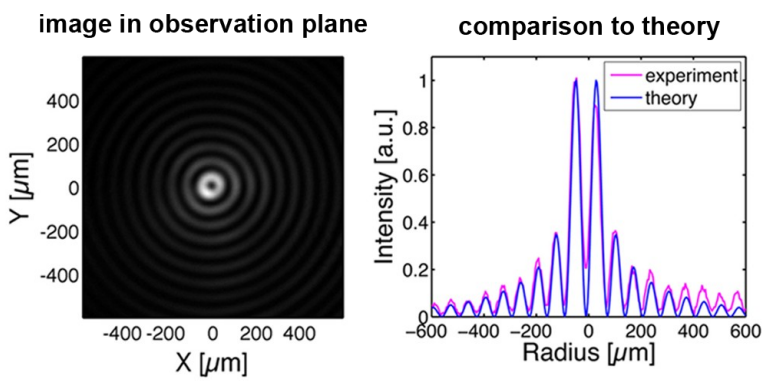

(b)

Fig. 2.: (a) schematic of the experimental setup, (b) image of the radially polarized Bessel-Gauss beam in the observation plane.

In conclusion, we obtained expressions for radially polarized Bessel-Gauss beams in ABCD optical systems. The form of the solution is experimentally verified by employing a fiber-based mode-converter. The expressions are useful for the setting up of optical systems for radially polarized Bessel-Gauss beams. Applications, include vacuum electron acceleration, imaging, and manipulation and probing of micro particles.

[1] S. Quabis, R. Dorn, M. Eberler, O. Glöckl, and G. Leuchs, "Focusing light to a tighter spot," Opt. Commun. 179, 1-7 (2000).

[2] L. Novotny, et al "Longitudinal Field Modes Probed by Single Molecules," Phys. Rev. Lett. 86, 5251-5254 (2001).

[3] L. J. Wong et al "Direct acceleration of an electron in infinite vacuum by a pulsed radially-polarized laser beam," Opt. Expr.18, 25035 (2010).

[4] Q. Zhan, "Trapping metallic Rayleigh particles with radial polarization," Opt. Express 12, 3377-3382 (2004).

[5] D. N. Schimpf, et al "Generalizing higher-order Bessel-Gauss beams: analytical description and demonstration," Opt. Expr. 20, 26852 (2012). 
[6] S. Ramachandran, et al "Generation and propagation of radially polarized beams in optical fibers," Opt. Lett. 34, $2525-2527$ (2009). 